\title{
REGÍMENES CAMBIARIOS DE IURE Y DE FACTO. EL CASO DE LA PESETA/DÓLAR, 1965-1998*
}

\author{
FRANCISCO J. LEDESMA RODRÍGUEZ \\ Universidad de La Laguna \\ MANUEL NAVARRO IBÁÑEZ \\ Universidad de La Laguna \\ JORGE V. PÉREZ RODRÍGUEZ ${ }^{b}$ \\ Universidad de Las Palmas de Gran Canaria \\ SIMÓN SOSVILLA RIVEROc \\ FEDEA y Universidad Complutense de Madrid
}

\begin{abstract}
RESUMEN
En este trabajo se aplican tres procedimientos estadísticos alternativos al tipo de cambio peseta/dólar norteamericano, con el objetivo de conocer el verdadero régimen cambiario al que estuvo sujeto durante el período 1965-1998. El estudio toma la perspectiva que señala la divergencia entre regímenes cambiario de facto y de iure. Los resultados indican que la peseta, al igual que otras monedas, no exhibió el régimen flotante que de iure tenía en relación al dólar durante las tres últimas décadas de su existencia. Por el contrario, el tipo de cambio peseta/dólar se movió dentro de un corredor más bien estrecho en dichos años.

Palabras clave: régimen cambiario, bandas de fluctuación, tipos de cambio fijos y flotantes, tipo de cambio peseta/dólar

* Los autores reconocen el apoyo financiero del Ministerio de Educación y Ciencia (Plan Nacional I+D: SEJ-2005 09094/ECON). Agradecemos los comentarios de un evaluador anónimo, así como de los participantes en el VIII Encuentro de Economía Aplicada.

a Departamento de Análisis Económico e Instituto Universitario de Desarrollo Regional, Facultad de Ciencias Económicas y Empresariales, Campus de Guajara, 38071 La Laguna, Tenerife, España. fledesma@ull.es; mnavarro@ull.es

b Departamento de Métodos Cuantitativos en Economía y Gestión, Facultad de Ciencias Económicas y Empresariales, Campus Universitario de Tafira, 35017 Las Palmas de Gran Canaria, España.jvperez@dmc.ulpgc.es

c FEDEA, Jorge Juan 46, 28001 Madrid. simon.sosvilla@fedea.es
\end{abstract}




\begin{abstract}
In this paper we apply three different statistical procedures to the peseta/dollar exchange rate with the aim of discovering the true foreign exchange regime followed by the monetary authorities during the 1965-1998 period. The study's perspective emphasizes the divergence between de jure and de facto exchange regimes. The results seem to imply that the peseta, as well as other currencies, did not exhibit the floating regime that de jure had in relation to the US dollar in the last three decades of its existence. On the contrary, the peseta/dollar exchange rate moved within rather narrow fluctuation bands during those years.
\end{abstract}

Keywords: Foreign exchange regime, fluctuation bands, fixed and floating exchange rates, peseta/dollar exchange rate

JEL Classification: F31; F33.

En este trabajo se aplican algunos procedimientos estadísticos a la historia del tipo de cambio de la peseta con el objetivo de analizar el verdadero régimen cambiario al que habría estado sujeta, al menos a partir de su inserción internacional dentro del marco institucional derivado de la Segunda Guerra Mundial. El estudio se enfoca desde la perspectiva propuesta por la literatura más reciente, en la que se hace hincapié en la divergencia entre regímenes cambiarios de iure y de facto, es decir, entre lo que declaran oficialmente las autoridades monetarias al Fondo Monetario Internacional (FMI) y lo que en la práctica aplican en sus actuaciones en los mercados de divisas.

Una vez que, con el Plan de Estabilización de 1959, se puso punto final a la última parte (1948-1959) de la época autárquica -caracterizada por un sistema de tipos de cambio múltiples-, el gobierno español acabó aceptando la unificación del tipo de cambio de la peseta. Así, después de su devaluación hasta las 60 pesetas/dólar, se estableció la convertibilidad de la divisa española dentro del régimen general de paridades fijas, aunque ajustables, establecido en Bretton Woods. Éste será, a partir de ese momento, el sistema cambiario que se mantendrá hasta su definitivo colapso en los primeros años de la década de 1970, siendo sustituido entonces -al menos de manera oficial- por un régimen de flotación que durará prácticamente, y bajo diversas vicisitudes, hasta el ingreso de la peseta en el Mecanismo de Cambios e Intervención (MCI) del Sistema Monetario Europeo (SME) el 19 de junio de 1989.

Este nuevo compromiso supranacional, que se insertaba en la perspectiva de la incorporación de España al proceso de la Unión Económica y Monetaria Europea (UEM), devolvía oficialmente a la peseta a un tipo de cambio semifijo ${ }^{1}$ con

\footnotetext{
${ }^{1} \mathrm{La}$ peseta se acogió a la posibilidad de, una vez fijada la paridad central, fluctuar dentro de una banda de \pm 6 por cien.
} 
respecto a las principales monedas europeas. La intención de las autoridades económicas era abiertamente la de lograr una mayor disciplina en el control de las variables monetarias, aceptando el ancla del SME, sobre todo del marco alemán, para acceder a la credibilidad antiinflacionaria del Bundesbank. Las contrapartidas más evidentes de este beneficio se encontraban tanto en la pérdida de autonomía en la elaboración de la política monetaria interna, por parte del Banco de España, como en la necesidad de controlar el déficit presupuestario, por parte del Gobierno. El objetivo último, la plena incorporación a la UEM, se cumplió con la desaparición definitiva de la peseta y su sustitución, como unidad de cuenta, por el euro el 1 de enero de 1999.

El trabajo se organiza de la siguiente manera: en el apartado 1 se ofrece una sucinta relación de los regímenes cambiarios en España y se discuten algunas de las razones por las que se han cuestionado recientemente los estudios llevados a cabo sobre regímenes cambiarios cuando se basaban en la clasificación oficial del FMI. El apartado 2 presenta las principales aproximaciones técnicas propuestas con la intención de conocer el verdadero régimen cambiario que tiene un país, así como su aplicación al tipo de cambio peseta/dólar en el período 1965-1998. Por último, en el apartado 3 se recogen las conclusiones más sobresalientes del análisis llevado a cabo.

\section{REGÍMENES CAMBIARIOS EN LA RECIENTE HISTORIA DE ESPAÑA}

La exclusión de España del Plan Marshall llevó a sus autoridades económicas a poner en marcha, en diciembre de 1948, un complejo régimen de tipos de cambio múltiples, que seguirá siendo en esencia un sistema de controles -extremadamente rígido- tanto de las transacciones comerciales como de las operaciones financieras con el exterior ${ }^{2}$. Las diversas modificaciones que se fueron introduciendo en este complejo régimen a lo largo de su período de vigencia trataron de simplificarlo, lo que llevó consigo la aceptación, a partir de 1950, de tipos de cambio más variables que fueron acomodándose de alguna manera a lo que ocurría en el mercado libre. Cada corrección introducida por las autoridades llevaba implícita, además, una importante devaluación de la peseta, aunque no se llegó en ningún caso a eliminar la sobrevaloración acumulada por ésta, generada en realidad por la muy distinta evolución de los precios entre España y el exterior ${ }^{3}$.

${ }^{2}$ Vid. Serrano Sanz (2000a). De hecho, la necesidad de autorización previa, y de la consiguiente concesión de licencias para realizar exportaciones o importaciones (o cualquier pago en moneda extranjera), hizo que en el mercado de divisas continuaran asignándose éstas vía cantidades (permitidas) y no por su precio. Véase también Martínez Ruiz (2001).

${ }^{3}$ La peseta se depreció desde las 13,63 pesetas por dólar en 1940 (cotización media en el mercado de Tánger) hasta las 60 pesetas/dólar que fijó el Plan de Estabilización de 1959. Véanse Alcaide Inchausti y Alcaide Guindo (2004), así como Serrano Sanz y Asensio (1997). 
La estrategia autárquica seguida por el gobierno franquista significó la total división entre las vertientes interior y exterior de la política monetaria. Ello se plasmó, por una parte, en la creación del Instituto Español de Moneda Extranjera (IEME) como encargado de la administración de las -cada vez más escasas- divisas, al que se hizo depender del Ministerio de Comercio, separando así al Banco de España -a su vez, totalmente subordinado a las decisiones del Ministerio de Hacienda- de la elaboración y gestión de la política cambiaria. Por su parte, a este último se le convirtió en un obsequioso suministrador de los recursos líquidos necesarios para el mantenimiento de una política monetaria que se puso completamente al servicio de la inflacionista política presupuestaria ${ }^{4}$.

La unificación definitiva del tipo de cambio se llevó a cabo con el Plan de Estabilización de 1959. Aun así, la política monetaria exterior permaneció en manos del IEME, separándola del control monetario interno, que seguía básicamente las directrices emanadas del Ministerio de Hacienda. El Banco de España continuó siendo un observador pasivo, ya que las reformas llevada a cabo durante la década de 1960 no incluyeron la puesta en marcha de un verdadero banco central, pues no se le concedieron los instrumentos imprescindibles para ejercer la labor propia de control de los agregados monetarios. Por ello, cuando la continua inflación diferencial española, consecuencia obligada de una política monetaria muy permisiva, terminó con los beneficios obtenidos a partir de las medidas estabilizadoras puestas en marcha en 1959, la apreciación de la peseta produjo un continuo deterioro del sector exterior que sólo podía resolverse mediante la devaluación, como así sucedió en noviembre de $1967^{5}$.

La caída del sistema de Bretton Woods en los primeros años de la década de 1970, y la consiguiente volatilidad de los mercados financieros, no tuvo como consecuencia inmediata la instauración de un régimen generalizado de tipos de cambio flexibles, como parecía esperable. Por el contrario, numerosos países relacionaron el valor externo de sus monedas con las divisas fuertes -o con una cesta de varias monedas- con las que se relacionaban de manera más intensa, persuadidos de que una mayor flexibilidad produciría una excesiva fluctuación cambiaria $^{6}$. Esta volatilidad era no sólo fuente de inestabilidad macroeconómica, sino que también acababa afectando negativamente a la inversión productiva, a los flujos comerciales internacionales y, por ende, al crecimiento económico. Así, numerosos países ligaron sus monedas al dólar estadounidense.

La mayor integración entre las políticas monetaria y cambiaria que pudo realizar el Banco de España a partir de 1973, con la asunción de las funciones del ya por entonces extinto IEME, le permitió hacer frente al término de la época de Bretton

\footnotetext{
${ }^{4}$ Vid. Martínez Ruiz (2000).

${ }^{5}$ Aixalá Pastó (2000).

${ }^{6}$ La creación de la «serpiente monetaria europea» en 1973 pretendía reducir las fluctuaciones monetarias entre los países miembros del Mercado Común Europeo.
} 
Woods $^{7}$ y al comienzo de la flotación de la peseta en 1974. Una flotación que, al decir de algún estudioso, pretendía lograr la estabilidad cambiaria de la peseta tomando como referencia, en un primer momento, un índice del tipo de cambio nominal frente a los países desarrollados y, desde 1986, otro indicador frente a las principales monedas comunitarias ${ }^{8}$. Por otra parte, la aparente intratabilidad del déficit presupuestario suponía un aumento continuo e incontrolado de la cantidad de dinero, al que el banco central deseaba poder atajar. Este período fue también el del final de la época franquista y el comienzo de la transición política hacia un sistema democrático, exhibiendo al mismo tiempo dos importantes devaluaciones de la peseta con respecto al dólar en los años de 1976 y de 1977, del 11 por cien y el 20 por cien respectivamente.

La segunda subida del precio del petróleo en 1979 y la consiguiente recesión europea provocaron en España una situación de crisis económica, que perduraría hasta mediados de la década siguiente. La política monetaria seguida por las autoridades hasta 1982 fue muy acomodaticia para reducir las tensiones políticas y financiar el creciente déficit presupuestario. La etapa abierta por el gobierno socialista, que comenzó en diciembre de 1982 con una devaluación de la peseta con respecto al dólar de un 7,6 por cien, exhibió por el contrario una mayor estabilidad política, lo que permitió un nuevo ajuste de la economía española con una política monetaria más restrictiva, aunque siempre lastrada por la inflacionaria financiación del déficit presupuestario público ${ }^{9}$.

La elección del régimen cambiario más adecuado para un país buscaba, durante la década de 1980, encontrar el sistema que diera la mayor protección teórica ante posibles perturbaciones monetarias y reales, proporcionando ante todo, de esta manera, un buen instrumento de estabilización. Por otra parte, la inestabilidad monetaria tan extendida durante estos mismos años exigió políticas nacionales de deflación y de consolidación presupuestaria, por lo que no es de extrañar que fueran los aspectos monetarios los que atrajeran prioritariamente la atención de los estudiosos ${ }^{10}$. En particular, con la fijación del tipo de cambio que llevaron a cabo algunos países se esperaba controlar la expansión monetaria interna, induciendo desde el exterior una mayor disciplina en las finanzas públicas ${ }^{11}$. La elec-

\footnotetext{
${ }^{7}$ El aumento del precio del petróleo, a finales de 1973, fue probablemente el principal problema económico al que tuvo que enfrentarse la peseta durante el primer lustro de la década de 1970. Todo ello iba unido a las tensiones políticas derivadas del fin del franquismo (Serrano Sanz, 2000b). De hecho, la crisis energética y el cambio político produjeron en general una fuerte depreciación de la peseta, alcanzando el tipo de cambio las 170 pesetas por dólar en 1985 (Alcaide Inchausti y Alcaide Guindo, 2004).

${ }^{8}$ Véase Serrano Sanz (2000b).

9 Ibidem.

${ }^{10}$ Vid. al respecto Levy-Yeyati y Sturzenegger (2001).

${ }^{11}$ Alberola y Molina (2004) han mostrado que las mayores posibilidades de financiación a corto plazo de los déficit presupuestarios bajo un sistema de tipos de cambio fijos o semifijos tienden a relajar el control fiscal, erosionando la sostenibilidad del régimen y el abandono a medio plazo del ancla cambiaria.
} 
ción entre la estabilidad de precios o la del tipo de cambio como objetivos intermedios se hizo en un contexto de escepticismo, muy justificado, hacia las posibilidades de control interno y de creciente confianza en las posibilidades de la estabilidad externa.

El nivel de credibilidad de los compromisos monetarios y cambiarios de un país dependía del prestigio antiinflacionario de sus autoridades económicas, lo que significaba para la mayoría de los países -con un historial escasamente edificante- la autoexigencia de fijar el tipo de cambio a una moneda fuerte y con suficiente reputación ${ }^{12}$. Así, las autoridades económicas, dada la creciente vinculación a Europa de la economía española, sobre todo desde la incorporación de ésta a la Comunidad Económica Europea en 1986, decidieron en 1989 la entrada de la peseta en el SME. De esta manera, y como era previsible al menos desde 1985, el marco alemán se convertía en la verdadera ancla de la política cambiaria de España.

Uno de los principales argumentos que se habían utilizado en la puesta en marcha del SME en 1979, y que también fue muy empleado por las autoridades monetarias españolas, fue precisamente que facilitaba los procesos de deflación y que elevaba los costes de ser permisivos con la inflación, forzando a sus asociados a comportarse responsablemente ${ }^{13}$. El SME proporcionaba la disciplina necesaria, asegurando que los déficit fiscales no serían en ningún caso apoyados con expansiones monetarias. En este contexto, Alemania aparecía naturalmente como el ancla más creíble con respecto a las expectativas monetarias y cambiarias de los países participantes. De hecho, el SME puede concebirse como un acuerdo institucional que ha permitido a sus miembros tomar prestada la reputación del Bundesbank, mediante la fijación de sus tipos de cambio al marco alemán ${ }^{14}$. De este modo, las monedas débiles conseguían un mayor grado de credibilidad por la existencia de efectos de reputación dentro del SME - es decir, que los países que tenían experiencias de inflación elevada utilizaron el sistema para obtener la credibilidad antiinflacionaria del Bundesbank ${ }^{15}$.

${ }^{12}$ Como han argumentado Bordo y Rockoff (1996), también en el período de 1870 a 1914 los países emergentes que pudieron hacerlo (Canadá, Australia y los Estados Unidos) se adhirieron al patrón oro, otros lo intentaron en ocasiones (Argentina, Brasil y Chile), mientras que algunos (Italia, España y Portugal) seguían políticas monetarias que trataban de conformarse a dicho sistema. Y lo hicieron, de acuerdo con estos autores, precisamente para enviar una señal de rectitud financiera a los mercados de capitales de los países más desarrollados.

${ }^{13}$ Martín Aceña (2000).

${ }^{14}$ El SME puede verse también como una regla monetaria que comprometía a las autoridades monetarias a seguir en el futuro una política que asegurase la estabilidad de precios. Cualquier desviación, provocada por alguna emergencia o perturbación, sería corregida mediante las adecuadas políticas antiinflacionistas. Con esta misma perspectiva, Bordo y Kydland (1995) han analizado el sistema clásico del patrón oro durante el período previo a la Primera Guerra Mundial.

${ }^{15}$ Para un estudio, en el que se toma al marco alemán como ancla, de la credibilidad cambiaria del Sistema Monetario Europeo a lo largo de toda su historia, ver Ledesma-Rodríguez et al. (2005a). 
En los años 90 se produjo de nuevo un cambio de actitud. Lo ocurrido durante la década, esto es, las presiones especulativas que experimentaron algunos de los países miembros del SME, entre ellos España, así como el desarrollo de las diversas crisis padecidas en Latinoamérica, Asia y Rusia, hizo que, una vez más, se pusiera en cuestión la oportunidad de un régimen cambiario fijo. De nuevo, el sistema más adecuado -en términos de su asociación con una mayor tasa de crecimiento real, una menor inflación y una más reducida volatilidad del output y de los precios- parecía ser ahora uno de libre flotación; eso sí, complementado con la persecución de algún objetivo moderado y creíble de inflación. Este cambio se produjo por la importancia que, en la década de 1990, alcanzaron los flujos de capital privado entre países, modificando seriamente el sistema financiero internacional. De esta manera, las crisis financieras de estos años se centraron sobre todo en la evolución de la cuenta de capital, a diferencia de lo que ocurría durante la década anterior, cuando predominaron las causas internas más tradicionales déficit fiscales elevados, monetización de los mismos, distorsiones en los mercados de bienes y factores productivos, entre otras ${ }^{16}$.

El caso de España puede tomarse como un ejemplo paradigmático de lo acaecido en la década de 1990 y, en particular, de lo limitado de la estabilidad externa sin acompañamiento de la política presupuestaria y de reformas estructurales en los mercados de bienes y de factores productivos, lo que impidió doblegar las expectativas de los agentes económicos. Así, la incorporación al MCI con un tipo de cambio peseta/marco sobrevaluado y una política fiscal procíclica en un contexto expansivo provocó continuas entradas de capital, un incremento imparable de las reservas ${ }^{17}$ y la consiguiente presión apreciadora sobre la peseta. Ésta se transformó en una de las divisas más fuertes del SME, ayudada por los altos tipos de interés fijados por el Banco de España, con una política suavemente contractiva que intentaba reducir la elevada inflación -el 6 por cien en promedio durante el período 1989-1992. La política monetaria se mostró insuficiente para depreciar la peseta en términos reales y bajar los tipos de interés ${ }^{18}$.

No es de extrañar que la tormenta monetaria desatada en septiembre de 1992 provocara hasta tres devaluaciones de la peseta, ya que los ataques especulativos contra el régimen de paridades fijas del MCI se centraron en aquellas monedas que exhibían unos fundamentos económicos más débiles. La crisis del SME sólo pudo cerrarse con la ampliación de las bandas de fluctuación en agosto de 1993 hasta un \pm 15 por cien, cuando ya la peseta se había depreciado en un 20 por cien en términos nominales en relación al marco. La política monetaria pudo disfrutar entonces de un cierto margen de maniobra para que el Banco de España se

\footnotetext{
${ }^{16}$ Summers (2000).

${ }^{17}$ Las reservas ascendían a 72.000 millones de dólares en junio de 1992.

${ }^{18}$ Gadea Rivas (2000).
} 
planteara la disminución de la inflación, dentro del cumplimiento de los criterios de convergencia nominal que llevarían a la UME. En la persecución de este objetivo, el Banco se vio ayudado por un creciente rigor fiscal que logró reducir el déficit presupuestario.

La lección que se extrajo a finales de la década de 1990 consistía en que, en un contexto de desregulación financiera y creciente liberalización de los movimientos de capital, ya no era posible fijar el valor externo de una moneda en términos de una divisa fuerte (o de una cesta de monedas). Se esperaba, por el contrario, que un tipo de cambio fijo aumentase la volatilidad del output real y acentuase el efecto negativo de las perturbaciones externas financieras y reales, al elevar las expectativas de obtención de ganancias especulando en contra del mantenimiento del tipo de cambio de una moneda en los mercados de divisas.

Algunos autores, comenzando con Bean (1992), antes incluso de las sucesivas crisis del SME en 1992-1993, y Eichengreen (1994) con posterioridad, han argumentado que, en un contexto de desregulación financiera y eliminación de las restricciones a los movimientos de capital, los regímenes cambiarios «intermedios» no son sostenibles a largo plazo por su fragilidad ante ataques especulativos ${ }^{19}$. Por ello, algunos investigadores afirman que la única elección posible se encuentra entre un tipo de cambio completamente fijo (e irrevocable), esto es, una unión monetaria -como la llevada a cabo por 12 países de la Unión Europea, con la creación del euro-, y un tipo de cambio totalmente flotante. Esta hipótesis de la «desaparición del régimen intermedio», como la denominó Frankel $(1999)^{20}$, implica que, ante el crecimiento espectacular de los movimientos internacionales de capital, la única solución viable a largo plazo parece hallarse en los extremos ${ }^{21}$.

La elección, que todo país ha de realizar en cualquier caso, entre un sistema u otro de tipos de cambio, ha llevado a la discusión empírica a centrarse también en la adecuada estimación de los costes y beneficios económicos de los diversos regímenes cambiarios. Una dificultad previa que ha surgido a menudo ha sido la gran variedad de éstos, ya que no existen únicamente dos posibilidades extremas -como parecía ser por los estudios que, naturalmente, empleaban la clasificación oficial del FMI-, sino que, por el contrario, los países utilizan en realidad una amplia

\footnotetext{
${ }^{19}$ El argumento de la «trinidad imposible», como lo ha denominado Fischer (2001), hace hincapié en la idea de que ninguna economía puede tener simultáneamente libertad de movimientos de capital, un tipo de cambio fijo y una política monetaria dirigida exclusivamente a objetivos nacionales.

${ }^{20}$ Frankel (1999) se mostraba en desacuerdo con esta idea. Una exposición matizada de un defensor de la «perspectiva bipolar» se encuentra en Fischer (2001). Para una reciente argumentación desfavorable a la «teoría de la exclusión del [régimen] intermedio», véase Reinhart y Reinhart (2003), donde se afirma que dicha hipótesis es una falacia «contraria a la teoría y a la evidencia». La mayoría de los países parece tener de facto un régimen cambiario intermedio.

${ }^{21}$ Eichengreen (1994) y (2000); Fischer (2001).
} 
variedad de sistemas cambiarios, tales como cajas de conversión (currency boards), bandas de fluctuación móviles o reptantes, y flotación sucia.

Más recientemente se ha señalado otra dificultad en la evaluación empírica del régimen cambiario: los países no parecen haber declarado el verdadero sistema que empleaban. La política cambiaria seguida de facto por numerosos Estados se ha revelado como muy alejada de lo que de iure afirmaban sus autoridades. Así, se ha mostrado que la mayoría de los gobiernos que declaran tener un régimen de flotación -como la peseta a partir de 1974- siguen en realidad otro sistema distinto, generalmente alguna forma de tipo de cambio fijo o semifijo, ya que muestran un considerable «miedo a la flotación»» ${ }^{22}$.

Este miedo a la libre flotación se debe, en general, a una elevada exposición al riesgo del tipo de cambio por estar tanto las administraciones públicas como las empresas endeudadas en divisas ${ }^{23}$. Unas expectativas desfavorables sobre la futura evolución del tipo de cambio suelen ir asociadas a modificaciones desestabilizadoras en los compromisos de los «inversores» con un país, produciendo salidas de capital y, lo que es peor, efectos de contagio en otras naciones. Por ello, estos estados no permiten que sus tipos de cambio se muevan libremente. Por el contrario, utilizan las reservas de divisas y los tipos de interés para mantener los movimientos del tipo de cambio dentro de aquellos límites que consideran más adecuados.

Una consecuencia de esta divergencia entre los «hechos» y las «palabras» de las autoridades monetarias ${ }^{24}$ ha sido la puesta en cuestión de los resultados obtenidos por los numerosos estudios empíricos que utilizaron la clasificación oficial elaborada por el FMI a partir de lo declarado por dichas autoridades. Por ello, antes de profundizar en la evaluación de los distintos regímenes cambiarios, se hace necesario que previamente se disponga de una clasificación de los regímenes de facto que sustituya a la que de iure han seguido hasta hace pocos años los estudiosos del tipo de cambio de la peseta.

En el trabajo que aquí se presenta se busca identificar la existencia de regímenes cambiarios implícitos ${ }^{25}$ en diversas épocas de la historia reciente de la peseta. Así, mediante la utilización de diversos procedimientos estadísticos que han sido

\footnotetext{
${ }^{22}$ Reinhart (2000); Calvo y Reinhart (2002), y Reinhart y Rogoff (2004).

${ }^{23}$ Los «inversores» no residentes suelen prestar únicamente en la moneda de su propio país, por lo que los residentes tienen que endeudarse en divisas, exponiéndose así a todas las modificaciones que sufra el tipo de cambio.

${ }^{24}$ En expresión de Levy-Yeyati y Sturzeneger (2000).

25 Se ha detectado en algunos estudios recientes que los bancos centrales han establecido bandas implícitas de fluctuación de sus monedas con respecto a alguna otra, y ello ocurre incluso en el caso de los tipos de cambio que suelen utilizarse como ejemplo de flotación más libre. Así, Fischer (2001) ha argumentado la posibilidad de que haya operado en diversas ocasiones una zona objetivo entre los tipos de cambio del dólar, el euro (el marco, anteriormente) y el yen, aunque fuera de una manera informal y muy flexible.
} 
propuestos en la literatura, y como una primera ilustración, se hace un análisis de la posible existencia de bandas implícitas de fluctuación, «acordadas» por las autoridades monetarias para intervenir en el mercado peseta/dólar, a lo largo del período 1965-1998.

\section{IDENTIFICACIÓN DEL RÉGIMEN CAMBIARIO PESETA/DÓLAR}

\subsection{Datos}

En este apartado se presentan, en primer lugar, algunas de las aproximaciones propuestas, con la intención de conocer el régimen cambiario que tiene un país. A continuación, se lleva a cabo una aplicación al tipo de cambio peseta/ dólar, durante el período 1965-1998, con el objetivo de detectar la existencia de bandas implícitas de fluctuación, «acordadas» por las autoridades monetarias españolas.

La primera utilización empírica de la divergencia entre regímenes de iure y de facto la llevaron a cabo Ghosh et al. (1997), aunque sólo emplearon las nueve posibilidades de su clasificación «más fina» en el estudio de la robustez de los resultados -alcanzados previamente con la clasificación oficial del FMI en tres categorías: fijos, intermedios y flotantes ${ }^{26}$. Por su parte, Calvo y Reinhart (2002) y Reinhart (2000) mostraron, con datos mensuales de 39 países emergentes para el período 1970-1999, que la mayoría de aquellos gobiernos que anuncian tener un régimen flotante puro intervienen regularmente en los mercados de divisas con la finalidad de estabilizar el tipo de cambio. Por ello, estos autores afirmaban la existencia de una verdadera epidemia de «miedo a la flotación», rechazando rotundamente que los sistemas cambiarios fijos tendieran a desaparecer.

Las variables utilizadas son: (1) el tipo de cambio mensual peseta/dólar (al cierre del mercado). La fuente de datos es el Banco de España; (2) la base monetaria de España; (3) las reservas monetarias (excepto el oro monetario) de España, y (4) las reservas monetarias norteamericanas, en miles de millones de dólares, que se han obtenido del Fondo Monetario Internacional. El periodo abarca desde enero de 1965 hasta diciembre de 1998.

${ }^{26}$ Ghosh et al. (1997) consideraron cuatro regímenes fijos: en relación a una moneda; con respecto a los Derechos Especiales de Giro; con respecto a una cesta de monedas conocidas, y, por último, en relación a una cesta desconocida. El grupo intermedio consistía en sistemas cooperativos (como el SME), flotantes no catalogados y flotantes dentro de unas bandas predeterminadas. El último grupo se componía de flotantes sin unas bandas predeterminadas y flotantes puros. 


\subsection{Indicador de flexibilidad efectiva}

En un estudio de los factores determinantes en la elección de sistema cambiario, Hélène Poirson (2001) ha propuesto la utilización de un indicador de la flexibilidad exhibida en realidad por un régimen cambiario. Así, el índice de flexibilidad efectiva del tipo de cambio es una variable que compara los movimientos de las reservas de divisas, como medida más aproximada del grado de intervención de las autoridades en los mercados de divisas, con la volatilidad observada en el tipo de cambio nominal.

De esta manera, el grado de flexibilidad del tipo de cambio de facto de un país i, FLT, se mide mediante el cociente entre el valor absoluto medio de la depreciación mensual del tipo de cambio nominal, ME, y el valor absoluto medio de las variaciones mensuales en las reservas de divisas normalizadas por la base monetaria del mes anterior -lo que permite aproximar el impacto monetario de estas variaciones)- MR:

$$
\mathrm{FLT}=\frac{M E}{M R}=\frac{\sum_{0}^{11}\left|E_{t-k}-E_{t-k-1}\right| / E_{t-k-1}}{\sum_{0}^{11}\left|R_{t-k}-R_{t-k-1}\right| / H_{t-k-1}}
$$

donde $E_{t}$ es el tipo de cambio, $R_{t}$ son las reservas de divisas, excluyendo el oro monetario, y $H_{t}$ es la base monetaria.

Los valores que puede tomar FLT van desde 0, en el caso de una unión monetaria -no existe una moneda independiente-, cuando $\mathrm{ME}=0$, hasta infinito por la nula intervención de las autoridades, ya que entonces $\mathrm{MR}=0$. El indicador mostrará valores más reducidos cuando se interviene fuertemente con la finalidad de hacer frente a movimientos no deseados en los mercados de divisas, ya que MR será relativamente más grande que ME. Por el contrario, aquellos países que confían más en la libre actuación de dichos mercados tendrán un valor más elevado del índice de flexibilidad.

Una debilidad del indicador de flexibilidad efectiva se refiere a la medición del grado de intervención a través de las variaciones de las reservas. Dichas variaciones se ven afectadas también por las operaciones internacionales corrientes y por los movimientos de capitales en los que participan tanto los agentes privados como las administraciones públicas de un país. Además, cuando se interviene utilizando las reservas puede ser que se haga para afectar a varias monedas simultáneamente. En cualquier caso, estas limitaciones del indicador FLT están igualmente presentes en todos aquellos procedimientos que utilizan los tipos de cambio y las reservas de divisas. A mayor abundamiento, el índice FLT tiene la ventaja de mostrar gráficamente la posición real de una moneda en relación con un régimen cambiario totalmente fijo. 
Los resultados obtenidos utilizando el indicador de flexibilidad efectiva se presentan en el Gráfico 1. La proximidad al valor cero mide la cercanía a un sistema de tipos de cambio fijos, como puede observarse que ocurría en los años 60 y en los primeros años de la década de 1970, bajo el sistema de Bretton Woods, recogiéndose también la devaluación de noviembre de 1967, desde 60 a 70 pesetas/dólar, como el más alejado del valor nulo. El resto de los valores más elevados del índice se corresponden con las devaluaciones de la peseta en 1976, 1977 y 1982, todas ellas con respecto al dólar, así como las cuatro devaluaciones del período 1992-95, aunque éstas se llevaron a cabo dentro de la zona objetivo establecida por el SME y en el contexto modificado por la ampliación al 15 por cien de los límites de fluctuación del ERM. Por tanto, este indicador presenta distorsiones cuando las monedas están sujetas a devaluaciones, confundiendo los ajustes efectuados en un régimen cambiario fijo o semifijo con una mayor flexibilidad del tipo de cambio.

\section{GRÁFICO 1}

\section{ÍNDICE DE FLEXIBILIDAD EFECTIVA}

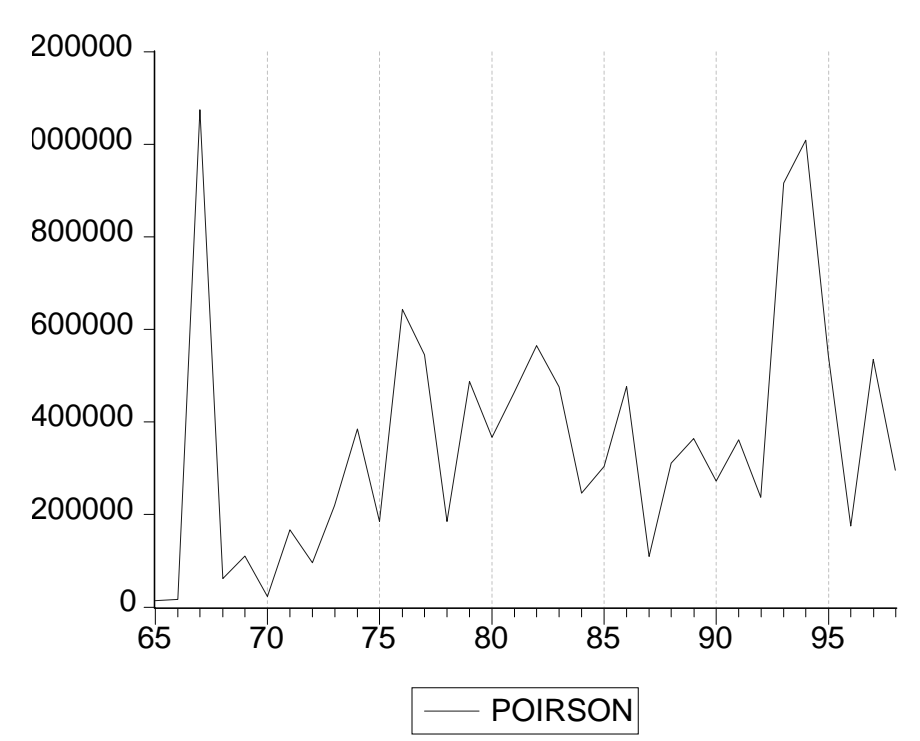

Nota: la proximidad al valor cero mide la cercanía a un régimen puro de tipos de cambio fijos. 
En cualquier caso, ha de señalarse que el indicador de flexibilidad efectiva alcanza los valores más elevados no únicamente por el cambio de la paridad, implícito en cualquier devaluación de la moneda nacional, ya sea con respecto al dólar o al marco, sino también por la menor intervención de las autoridades monetarias en el mercado de divisas cuando han decidido llevar a cabo la devaluación de su moneda -sobre todo si durante el período previo había existido presión especulativa en contra de la peseta.

\subsection{Identificación de sistemas cambiarios}

Coudert y Dubert (2003) han propuesto un procedimiento estadístico secuencial para identificar los regímenes cambiarios. Para ello, los separan en cinco categorías: libre flotación, flotación sucia, tipo fijo, tipo fijo-reptante y devaluación, con la finalidad de construir una clasificación trimestral a partir de los datos semanales de los tipos de cambio y mensuales de las reservas de divisas. La aplicación la hacen a una muestra de los diez principales países del sureste asiático durante el período 1990-2002.

La técnica utilizada se desarrolla en cinco etapas. En la primera, se calcula la tendencia anual de las series semanales del tipo de cambio. Si la tendencia es positiva (devaluación), las series sin tendencia se computan para distinguir entre sistemas fijos y flexibles. A continuación, a las series de tipo de cambio utilizadas se les elimina la tendencia en los períodos en que ésta se muestra positiva. Si, por el contrario, la tendencia es negativa (apreciación), ha de establecerse primero su significatividad, por lo que proponen que sea superior a un umbral del 2 por cien anual. En el caso de que lo sea, el régimen cambiario correspondiente no puede ser fijo, por lo que se pasa directamente a la cuarta etapa, en la que se examina si la flotación es pura o sucia. Cuando el valor sea inferior a ese 2 por cien, será necesita pasar a la segunda etapa, ya que entonces se estará ante un sistema fijo o uno flexible sin tendencia.

En la segunda etapa se comparan las varianzas trimestrales de las series a estudiar del tipo de cambio -que pueden estar sin tendencia, dependiendo del resultado de la etapa 1- con las de las monedas de referencia (por definición, flotantes) ${ }^{27}$.

${ }^{27}$ Se supone que el cociente de las varianzas sigue una distribución chi-cuadrado con n-1 grados de libertad, siendo n el tamaño de la muestra:

$$
(n-1) \frac{\hat{\sigma}^{2}}{\sigma_{0}^{2}} \sim \chi_{(n-1)}^{2}
$$

Se lleva a cabo un contraste de igualdad de varianzas al 5 por cien de significación, donde $\sigma_{0}^{2}$ es la media de las variaciones trimestrales de las principales monedas flotantes y $\sigma^{2}$ es la varianza de la monedas asiáticas analizadas. Una vez realizado el contraste, se elabora una clasificación binaria inicial, donde las naciones con un régimen fijo son aquellas que exhiben una varianza estrictamente menor que la de los países de referencia. Siempre que no sea así, es porque han adoptado un régimen flexible. 
En la tercera etapa se estudia la tendencia determinística anual, obtenida en la primera etapa, con el fin de distinguir entre tipos fijos y tipos fijos-reptantes. A los primeros se les asignan las observaciones en las que la tendencia anual del tipo de cambio sea inferior al 2 por cien, mientras que, en otro caso, se las clasifica como propias de un régimen fijo-reptante.

En la cuarta etapa se calcula la varianza de la tasa de crecimiento de las reservas oficiales de divisas de las monedas clasificadas como flexibles en la primera etapa, con el objetivo de diferenciar entre episodios de flotación libre y episodios de flotación sucia ${ }^{28}$. De nuevo, se realiza un contraste de igualdad para, posteriormente, efectuar una comparación de las varianzas que desvirtúa el sentido estadístico de aquél.

Por último, en la quinta etapa se calculan las tendencias trimestrales determinísticas con el fin de detectar las posibles devaluaciones repentinas del tipo de cambio. Al analizar las naciones del sureste asiático, con un historial de depreciaciones moderadas -excepto en épocas de crisis-, se utiliza un umbral del 6 por cien.

En el Cuadro 1 se presentan los resultados obtenidos siguiendo la secuencia de contrastes para identificar el sistema cambiario de la peseta/dólar, aunque modificando los contrastes de igualdad en varianzas respecto al tipo de cambio de referencia (libra esterlina/dólar), que se supone representativo de la flotación libre. Se considera como hipótesis nula tanto el que las varianzas del tipo de cambio peseta/dólar sean mayores o iguales que las de la libra esterlina/dólar, para determinar la existencia o no de flotación pura, como el que las varianzas de las variaciones de las reservas sean mayores o iguales que las variaciones en las reservas de divisas de la Reserva Federal estadounidense ${ }^{29}$.

${ }^{28}$ Para obtener las varianzas trimestrales de las reservas se computan las varianzas mensuales sobre un período móvil de quince meses (los seis meses anteriores, el trimestre en cuestión y los seis meses siguientes). Suponiendo que estas variaciones mensuales exhiban una distribución normal, las varianzas trimestrales seguirían una distribución chi-cuadrado con catorce grados de libertad. Por ello, para aquellos países catalogados como flexibles en la segunda etapa, se lleva a cabo un contraste de igualdad de varianzas entre las varianzas trimestrales en las tasas de cambio de las reservas y las varianzas trimestrales promedio de las tasas de variación de las reservas de las monedas de referencia. Cuando las varianzas de las reservas son menores o iguales a las de referencia, se estará ante un régimen flotante mientras que si, por el contrario, son mayores, el sistema exhibe una flotación sucia.

${ }^{29}$ Formalmente, para comparar si la varianza del tipo de cambio peseta/dólar es mayor que otra de flotación libre se contrasta la siguiente hipótesis:

$$
\begin{aligned}
& H_{0}: \sigma^{2} \geq \sigma_{0}^{2} \\
& H_{1}: \sigma^{2}<\sigma_{0}^{2}
\end{aligned} \text {, donde } \sigma_{0}^{2} \text { es la varianza de la serie en flotación libre. }
$$

La región de aceptación de la hipótesis nula es: $(n-1) \frac{s^{2}}{\sigma_{0}^{2}} \geq \varepsilon$, siendo $n$ el tamaño muestral, $s^{2}$ la cuasivarianza muestral de la serie del peseta/dólar y el valor crítico de la distribución chi-cuadrado para $n-1$ grados de libertad al nivel de confianza del $1-\alpha$. El tamaño muestral para el cálculo de las varianzas es de 60 días (el equivalente a tres meses, considerando semanas de cinco días hábiles). 
Los resultados muestran el predominio de un régimen cambiario fijo en la gran mayoría de los trimestres del período muestral, comprendido entre los años de 1965 y 1998. La única devaluación captada como tal es la de noviembre de 1967, mientras que las devaluaciones del primer trimestre de 1976 y del último de 1982 se detectan como regímenes de flotación. El resto de las modificaciones de la paridad aparecen como tipos de cambio fijo o fijo-reptante.

\section{CUADRO 1}

CLASIFICACIÓN REGÍMENES CAMBIARIOS DE FACTO AL 95\%

\begin{tabular}{|c|c|c|c|c|}
\hline año $\backslash$ trimestre & I & II & III & IV \\
\hline R1965 & peg & peg & peg & peg \\
\hline R1966 & peg & peg & peg & peg \\
\hline R1967 & peg & peg & peg & devaluación \\
\hline R1968 & peg & peg & peg & peg \\
\hline R1969 & peg & peg & peg & peg \\
\hline R1970 & peg & peg & peg & peg \\
\hline R1971 & peg & peg & peg & flotación libre \\
\hline R1972 & flotación libre & peg & peg & peg \\
\hline R1973 & peg & peg & peg & peg \\
\hline R1974 & peg & peg & peg & peg \\
\hline R1975 & peg & peg & peg & peg \\
\hline R1976 & flotación libre & peg & peg & peg \\
\hline R1977 & crawling_peg & crawling_peg & crawling_pe & gcrawling_peg \\
\hline R1978 & peg & peg & flotación libre & peg \\
\hline R1979 & peg & peg & peg & peg \\
\hline R1980 & peg & peg & peg & peg \\
\hline R1981 & peg & peg & peg & peg \\
\hline R1982 & peg & peg & peg & flotación dirigida \\
\hline R1983 & peg & peg & peg & peg \\
\hline R1984 & peg & peg & peg & peg \\
\hline R1985 & peg & peg & peg & peg \\
\hline R1986 & peg & peg & peg & peg \\
\hline R1987 & peg & peg & peg & peg \\
\hline R1988 & peg & peg & peg & peg \\
\hline R1989 & peg & peg & peg & peg \\
\hline R1990 & peg & peg & peg & peg \\
\hline R1991 & peg & peg & peg & peg \\
\hline R1992 & peg & peg & peg & peg \\
\hline R1993 & crawling_peg & crawling_peg & crawling_peg & crawling_peg \\
\hline R1994 & peg & peg & peg & peg \\
\hline R1995 & peg & peg & peg & peg \\
\hline R1996 & peg & peg & flotación libre & peg \\
\hline R1997 & flotación libre & peg & flotación libre & peg \\
\hline R1998 & peg & peg & peg & $=p e g$ \\
\hline
\end{tabular}




\subsection{Bandas implícitas de fluctuación}

Reinhart y Rogoff (2004), en su extensa investigación, recogen la evolución histórica de los datos mensuales de los tipos de cambio de 153 países entre 1946 y 2001, haciendo hincapié en la existencia de mercados de divisas duales o múltiples, o incluso paralelos -sean legales o no-, lo que les lleva a distinguir hasta quince categorías cambiarias posibles.

En el caso de que no hubiera un mercado dual, para clasificar adecuadamente la situación cambiaria de facto, Reinhart y Rogoff (2004) basan su análisis en la variación porcentual del valor absoluto del tipo de cambio, así como en la probabilidad de mantenerse el mismo en una determinada banda -del 1 por cien, 2 por cien y 5 por cien- de fluctuación a lo largo de dos o cinco años rodantes ${ }^{30}$.

Con respecto a las bandas declaradas o de facto se sigue también un proceso en dos etapas. Si la banda ha sido explícitamente declarada por las autoridades y no existe un mercado paralelo, se la acepta, excepto en el caso en que hubiéramos encontrado durante el proceso anterior que nos hallamos ante un tipo de cambio fijo de facto. Reinhart y Rogoff (2004) también verifican si las bandas anunciadas (oficiales) y las reales coinciden, ya que las primeras suelen ser más anchas. A continuación, se calcula la probabilidad de que la variación del tipo de cambio se mantenga dentro de una banda de \pm 2 por cien en un período rodante de cinco años $^{31}$ : si dicha probabilidad es mayor o igual al 80 por cien, el régimen cambiario se cataloga como una banda estrecha, reptante o móvil durante el tiempo que se mantenga continuamente en ese 80 por cien.

En este trabajo se obtienen las bandas implícitas de fluctuación para el tipo de cambio peseta/dólar utilizando este procedimiento e incorporando una variante adicional. Así, en el Gráfico 2 se muestran los resultados alcanzados mediante la aplicación del procedimiento propuesto por estos autores, con el que se calcula la proporción de meses de los veinticuatro rodantes, incluyendo el actual, en los que la variación mensual porcentual del tipo de cambio es inferior al 1 por cien. Atendiendo al umbral del 80 por cien, únicamente habría un acercamiento a la presencia de bandas en las etapas más tempranas del periodo muestral, es decir, durante la época de Bretton Woods, así como poco después del abandono de dicho sistema, hasta 1979.

En el Gráfico 3 se hace lo mismo, pero considerando una banda de fluctuación del 2 por cien. Adoptando otra vez el umbral del 80 por cien, el procedimiento confirma la presencia de bandas entre los años 1965-1981, así como en 1985 y en

\footnotetext{
${ }^{30}$ La clasificación de Reinhart y Rogoff (2004), al centrarse únicamente en el estudio de los tipos de cambio, no considera las variaciones en las reservas oficiales de divisas, que indicarían las intervenciones de las autoridades.

${ }^{31}$ Cuando los límites de fluctuación declarados son más amplios, la verificación se realiza con una probabilidad de encontrarse en una banda de \pm 5 por cien.
} 


\section{GRÁFICO 2}

PORCENTAJE DE VARIACIONES MENSUALES

DENTRO DE BANDAS DEL 1\%

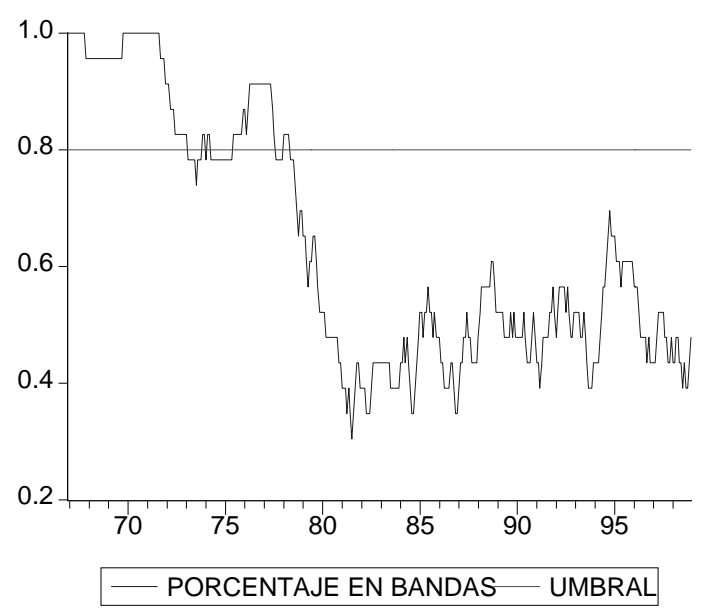

GRÁFICO 3

PORCENTAJE DE VARIACIONES MENSUALES

DENTRO DE BANDAS DEL 2\%

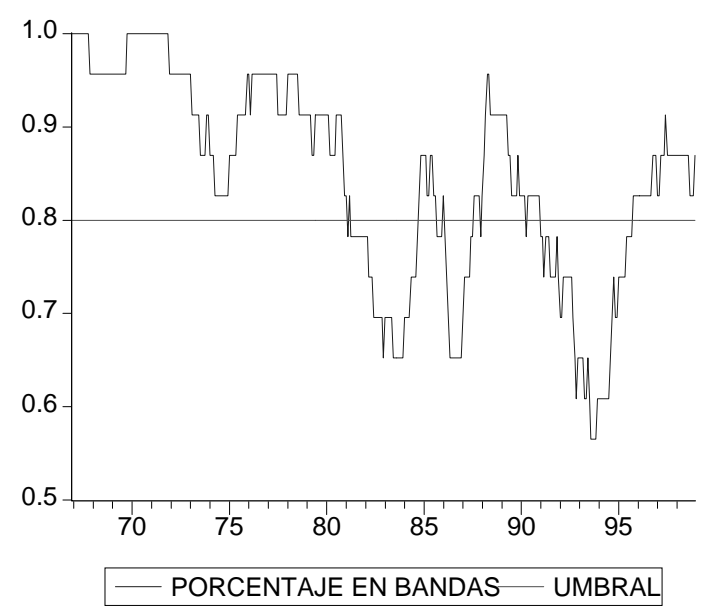




\section{GRÁFICO 4}

CONTRASTE SOBRE LAS VARIACIONES MENSUALES PROMEDIO DENTRO DE BANDAS DEL 1\%

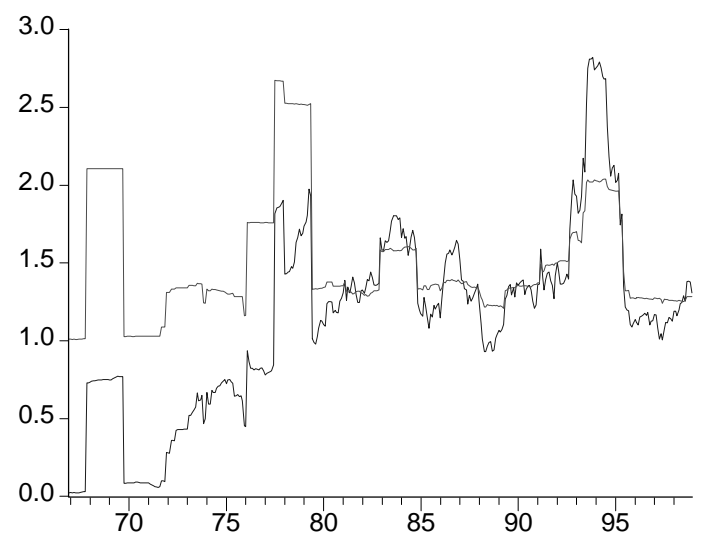

— PROMEDIO VARIACIÓN- - REGIÓN CRÍTICA

\section{GRÁFICO 5}

CONTRASTE SOBRE LAS VARIACIONES MENSUALES PROMEDIO DENTRO DE BANDAS DEL 2\%

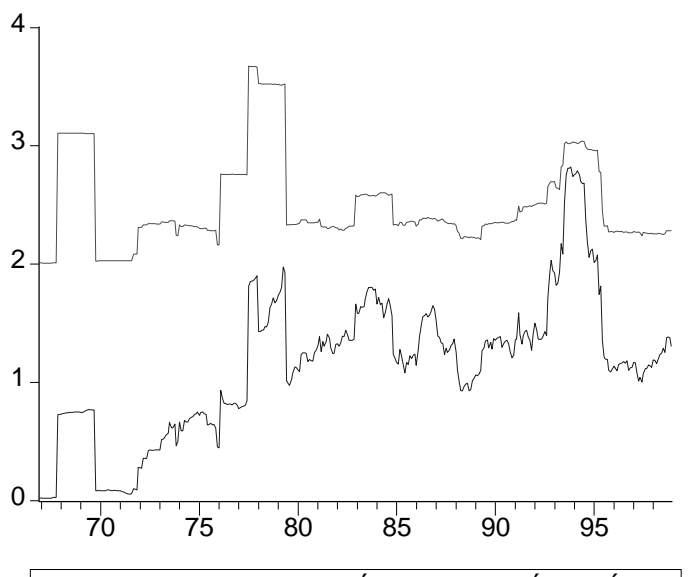

- PROMEDIO VARIACIÓN — REGIÓN CRÍTICA 
1989-90 -justo antes de la incorporación de España a la entonces Comunidad Económica Europea y al SME, respectivamente-, y en el período previo a la definitiva unión monetaria (1996-1999). Estos resultados sugieren que, tras la incorporación de la peseta al MCI, el dólar perdió importancia como moneda de referencia para la divisa española.

Una manera de mejorar la aproximación de Reinhart y Rogoff (2004) consiste en la contrastación directa de que las variaciones mensuales absolutas porcentuales promedio de cada periodo de veinticuatro meses sean significativamente inferiores a un 1 por cien o a un 2 por cien, como se explica detenidamente en Ledesma et al (2005b) ${ }^{32}$.

La aplicación de este procedimiento estadístico, sin recurrir previamente a contar periodos como se hace en Reinhart y Rogoff, se recoge en los gráficos 4 y 5 . Así, en el Gráfico 4 se presentan los resultados cuando se consideran unas bandas de fluctuación del \pm 1 por cien. En trazo oscuro aparece el valor medio -de veinticuatro meses rodantes- de las variaciones absolutas porcentuales del tipo de cambio de cada mes respecto al mes anterior, mientras que en trazo claro se representa la serie para la región crítica. Cuando la región crítica se encuentra por encima del promedio de las variaciones porcentuales significa que la hipótesis nula no se rechaza, sugiriéndose la aceptación de la presencia de bandas menores o iguales al \pm 1 por cien. Esto último ocurre casi siempre y especialmente durante el período 1965-1978, por lo que el contraste sugiere la existencia de bandas de fluctuación de \pm 1 .

Como puede observarse en el Gráfico 5, el promedio de las variaciones mensuales absolutas del tipo de cambio se encuentra en todo momento por debajo de la región crítica cuando se utiliza una banda del \pm 2 por cien, sugiriendo -dado el carácter rodante de los veinticuatro meses del contraste- que en la totalidad del periodo de estudio han existido unos corredores mensuales de fluctuación del 2 por cien.

\section{CONCLUSIONES FINALES}

Contrariamente a lo que parecía esperarse, después de la caída de Bretton Woods los tipos de cambio flotantes no acabaron imponiéndose. De hecho, los

${ }^{32}$ Para contrastar si la media poblacional (de las variaciones mensuales en períodos de veinticuatro meses) es menor o igual que una media $\mu_{0}( \pm 1 \%$ o $\pm 2 \%)$ determinada, el contraste adopta la siguiente forma:

$H_{0}: \mu \leq \mu_{0}$
$H_{1}: \mu>\mu_{0}$ , donde $\mu_{0}$ es la media determinada.

La región de aceptación de la hipótesis nula es $\bar{x} \leq \varepsilon$, siendo $\bar{x}$ la media muestral de la peseta/ dólar y $\varepsilon=\mu+t_{n-1,-\alpha} \frac{\sigma}{\sqrt{n}}$, donde $t_{n-1,1-\alpha}$ es el valor crítico de la distribución normal estándar al nivel de confianza del $\mathrm{I}-a, \sigma$ es la desviación muestral de la serie $y n$ el tamaño muestral. Al nivel de significación del 5 por cien se elige el valor crítico de 1,71. 
sistemas cambiarios fijos y semifijos parecen ser más la norma que la excepción. La historia reciente del tipo de cambio de la peseta durante los años de las décadas de 1970 y 1980 apoya ciertamente esta afirmación: manifiesto predominio de los regímenes cambiarios semifijos. Incluso durante la década de 1990 se sigue apreciando la inexistencia de libre flotación con el tipo de cambio peseta/dólar, y ello a pesar de la incorporación de la peseta al MCI.

Con la finalidad de probar estas argumentaciones, en la presente investigación se han utilizado tres aproximaciones alternativas sugeridas por la literatura más reciente. Uno de los resultados más importantes conseguidos es que los tres procedimientos dan lugar al mismo resultado: la peseta se movió dentro de un corredor más bien estrecho en los años posteriores al sistema de Bretton Woods. Parece clara, por tanto, la existencia de un cambio muy controlado por la autoridades monetarias españolas, ya que, temerosas de no poder gestionar la volatilidad cambiaria, mantuvieron de facto una fuerte conexión con la moneda norteamericana.

\section{BIBLIOGRAFÍA}

Aixalá PAstó, J. (2000): «De la estabilización a la crisis: la peseta en Bretton Woods, 1959-1973», en J. L. García Delgado y J. M. Serrano Sanz (dirs.), Del real al euro. Una historia de la peseta. Barcelona: Colección Estudios Económicos «la Caixa», Núm. 21, pp. 151-168.

Alberola, E. y Molina, L. (2004): «What does really discipline fiscal policy in emerging markets? The role and dynamics of exchange rate regimes». Madrid: Banco de España. Documento de Trabajo 0403.

Alcaide Inchausti, J. y Alcaide Guindo, P. (2004): «Evolución de la peseta en la historia (años 1868-2001)». Papeles de Economía Española 100, pp. 59-71.

BEAN, C. (1992): «Economic and Monetary Union in Europe». Journal of Economic Perspectives 6, pp. 101-121.

Bordo, M. D. y Kydland, F. E. (1995): «The Gold Standard As a Rule: An Essay in Exploration». Explorations in Economic History 32, pp. 423-464.

Bordo, M. D. y Rockoff, H. (1996): «The Gold Standard As a `Good Housekeeping Seal of Approval'». Journal of Economic History 56 (2), pp. 389-428.

Calvo, G.A. y ReInHART, C. M. (2002): «Fear of Floating». Quarterly Journal of Economics 117, pp. 379-408.

Coudert, V. y Dubert, M. (2004): «Does Exchange Rate Regime Explain Differences in Economic Results for Asian Countries?». París: Centre d’Etudes Prospectives et d’Informations Internationales (CEPII), Working Paper n²004-05.

EICHEngreen, B. (1994): International Monetary Arrangements for the $21^{\text {st }}$ Century. Washington, DC: The Brookings Institution.

-, (2000): «The EMS Crisis in Retrospect». Washington, DC: NBER Working Paper Series, Working Paper 8035, .

FischeR, S. (2001): «Exchange Rate Regimes: Is the Bipolar View Correct?». Journal of Economic Perspectives 15, pp. 3-24. 
Frankel, J. (1999): No Single Currency is Right for all Countries or at all Times, Princeton: Princeton University Press. Essays in International Finance, 215.

Gadea Rivas, M. D. (2000): «La peseta en la cultura de la estabilidad, 1989-1999», en J. L. García Delgado y J. M. Serrano Sanz (dirs.), Del real al euro. Una historia de la peseta. Barcelona: Colección Estudios Económicos «la Caixa», Núm. 21, pp. 169-190.

Ghosh, A. R., Gulde, A. M., Ostry, J.D. y Wolf, H. C. (1997): «Does the Nominal Exchange Rate Regime Matter?». Washington, DC: NBER Working Paper Series. Working Paper 5874.

Ledesma Rodríguez, F., Navarro Ibáñez, M., Pérez Rodríguez, J. y Sosvilla Rivero, S. (2005a): «Assessing the Credibility of a Target Zone: Evidence from the EMS». Applied Economics 37(19), pp. 2265-2287.

- (2005b): «Regímenes cambiarios de facto y de iure. Una aplicación al tipo de cambio yen/dólar». Moneda y Crédito 221, pp. 37-68.

Levy-Yeyati, E. y Sturzenegger, F. (2000): «Classifying Exchange Rate Regimes: Deeds vs. Words». Buenos Aires: Universidad Torcuato Di Tella. CIF Working Paper 02/2000.

- (2001): «Exchange Rate Regimes and Economic Performance». IMF Staff Papers 47, Special Issue, pp. 62-98.

Martín Aceña, P. (2000): El Servicio de Estudios del Banco de España 1930/2000. Madrid: Banco de España.

Martínez Ruiz, E. . (2000): «El control de cambios en la España franquista: el Instituto Español de Moneda Extranjera». Alcalá de Henares: Universidad de Alcalá de Henares. Tesis doctoral no publicada.

- (2001): «Sector exterior y crecimiento en la España autárquica». Revista de Historia Económica XIX, pp. 229-251.

Poirson, H. (2001): «How Do Countries Choose Their Exchange Rate Regime?». Washington, DC: International Monetary Fund. IMF Working Paper 01/46.

Reinhart, C. M. (2000): «The Mirage of Floating Exchange Rates». American Economic Review, Papers and Proceedings 90, pp. 65-70.

Reinhart, C. M. y Reinhart, V. R. (2003): «Twin Fallacies About Exchange Rate Policy in Emerging Markets». Moneda y Crédito 216, pp. 11-29.

Reinhart, C.M. y Rogoff, K. S. (2004): «The Modern History of Exchange Rate Arrangements: A Reinterpretation». Quarterly Journal of Economics 119, pp. 1-48.

Serrano Sanz, J. M. (2000a): «Veinte años de soledad. La autarquía de la peseta, 19391959», en J. L. García Delgado y J. M. Serrano Sanz (dirs.), Del real al euro. Una historia de la peseta. Barcelona: Colección Estudios Económicos «la Caixa», Núm. 21, pp. 107-124.

- (2000b): «Técnica sin disciplina en los años de flotación, 1974-1989», en J. L. García Delgado y J. M. Serrano Sanz (dirs.), Del real al euro. Una historia de la peseta. Barcelona: Colección Estudios Económicos «la Caixa», Núm. 21, pp. 151-168.

Serrano Sanz, J. M. y Asensio, M. J. (1997): «El ingenierismo cambiario. La peseta en los años del cambio múltiple, 1948-1959». Revista de Historia Económica XV, pp. 545-573.

Summers, L. (2000): «International Financial Crises: Causes, Prevention, and Cures». American Economic Review, Papers and Proceedings 90, pp. 1-16. 